\title{
Key observations in terms of management of electronic health records from a mHealth perspective
}

\author{
Varadraj P. Gurupur \\ School of Global Health Management and Informatics, University of Central Florida, Orlando, FL, USA \\ Correspondence to: Varadraj P. Gurupur. 528 W. Livingston Street, Suite 401, Orlando, FL 32801, USA. Email: varadraj.gurupur@ucf.edu.
}

\begin{abstract}
The article is a narrative review that briefly describes some of the recent advances in healthcare data management that will have positive effect on mHealth. The advances described in this article are in fact innovation introduced by the author to the field of data management with respect to electronic health records. The research delineated is transdisciplinary in nature and will potentially have positive impact on healthcare outcomes. Also, the article illustrates the necessity for an out of the box thinking approach to improve mHealth while discussing the current impending issues related to data incompleteness of electronic health records and the much-needed decision support systems for mHealth. It is to be noted that most of the electronic health records are now accessed by patients through mobile devices. These mobile devices will run as clients while much of the heavy computing is performed using servers. Here it is important to discuss some of the important technologies and methods used for decision making. The article attempts to present a discussion on how this myriad of intertwining technologies support this decision making with respect to electronic health records. More importantly it is these processes that assist in decision making and efficiency for both mHealth users and providers. In this respect, the article first provides insights on the complexities of decision making involved with electronic health records. This is followed by a discussion on the problem of data incompleteness of electronic health records. Finally, the author provides some insights into the gravity of the problem of data incompleteness in terms of revenue loss/gain for healthcare providers.
\end{abstract}

Keywords: Data incompleteness; decision support systems; electronic health records

Received: 14 September 2021; Accepted: 11 January 2022; Published: 20 April 2022.

doi: 10.21037/mhealth-21-39

View this article at: https://dx.doi.org/10.21037/mhealth-21-39

\section{Introduction}

My first inception to the healthcare industry started when I started working on pharmaceutical data for a large hospital back in 2005. This era was before lawmakers in the United States decided to implement the HITECH act. The healthcare industry at that time was in a state where they wanted to move forward with information technology; however, there was no clear motivation to do so. Many departments would use a combination of tools for performing different tasks or sometimes a task may be performed using a combination of several tools and other resources (1). Additionally, it is important to mention that telemedicine was many times delivered through a telephonic conversation largely due to the lack of high-speed internet and other infrastructures. This and other such situations created the need for data semantics and linking data for the purpose of decision support; thereby, reducing the need for certain expensive infrastructures of the yesteryears. It is to be noted that some these infrastructures are now more easily available compared to the year 2005 .

As the saying goes that the necessity is the mother of innovation; the needs of yesterday have led to the innovations of today. Decision support using semantic web-a concept introduced by Tim Berners Lee gained momentum in the early 2000s since there was plethora of data generated by the World Wide Web. Researchers and other stake holders wanted to exploit this situation for a wide variety of purposes. This was perhaps the beginning of a new thinking in healthcare information technology. This 
era led to the electronic health records being stored in the form of Resource Description Framework (RDF) and other eXtensible Markup Language (XML) formats. Here our research team while working on concept maps realized that concept maps could be converted into XML-based formats. Once the data is converted into such formats a suitable parser could be used to extract the required information for decision support. Let us keep in mind that this was only the first step. Further decision making needs a knowledge base, which leads to the question on how you could build a knowledge base. There were no clear answers to this difficult question. The other challenge here was that very few researchers ventured into this territory. Mainly because the chance of failure was extremely high. To add to this, it is a known fact that both government and private funders support the idea of feasibility of success while deciding to fund the project. This mindset was also found within private industry equipped with research departments. The change however manifested slowly with some early adapters such as companies that develop electronic health records capturing the ground while a few large organizations involved in software development underestimated the complexity involved in developing decision support systems for healthcare. However, many of these projects failed because the team members and especially the team leaders failed to comprehend the complexity of diseases and its relationship to human anatomy. This was at a time when the necessary infrastructure for such endeavors such as Unified Medical Language System (UMLS), and Bio2RDF were not fully available.

This situation created a necessity for infrastructure to be developed in healthcare informatics. Federal organizations in the United States such as National Library of Medicine got involved in making such infrastructure available to researchers. Although these initiatives were started much earlier in the 1980s their representation using semantic web was presented much later in 2000s. Although, the infrastructure was available in a non-usable form previously it got transformed into a more usable format.

\section{Current state of semantic networks for healthcare data management with electronic health records}

Fast forward to the present scenario, there is a need to advance decision support science using: (I) new domain knowledge structures to integrate available data with domain knowledge to form the required information; (II) new heuristics on the acquired information to form new knowledge; and (III) using this knowledge to generate the required recommendation by introducing new algorithms for decision support. Many times, domain knowledge is available to scientists and researchers in different formats such as database tables, XML-based documents, textual data, and semantic networks. In this situation, the key challenge would be to introduce, methods, and algorithms to capture the domain knowledge available in different forms and to transform it into a machine-actable form of information by integrating this transformed domain knowledge with available data $(2,3)$. To add to this, the information received from the aforementioned process by integrating domain knowledge and available data can be used to generate knowledge critical for decision support. This knowledge can be generated by applying the required heuristics (4-7) on the information generated. The form of heuristics applied here is that of representativeness described by Kahneman et al. (8). The term "knowledge" used here is considered with respect to active experimentation process described in (9).

Marewski and Gigerenzer (10) emphasized the need for and importance of heuristic decision making in medicine (11). They define heuristics as, "simple decision strategies that ignore part of the available information, basing decisions on only a few relevant predictors". They also indicate the fact that heuristics outperform traditional information centered methods such as regressions and traditional medical diagnosis. Based on these discoveries we can ascertain that heuristic decision making can play a vital role in decision making. The key challenge here will be translating the knowledge associated with heuristics into the decision support system. This is often achieved by translating this knowledge into machine-actable form of information (12). Using domain ontologies to represent this information to delineate a causal connection is an area of computation that may need further exploration $(13,14)$. Orgun and Meyer (13) state that, "semantic interoperability between ontologies is essential for enabling communication and sharing of information between heterogeneous systems". Also, decision support makes use of patient data available in a machine-actable form. The strength of data completeness $(15,16)$ is critical in this context. Majeed et al. (15) comment that, "the completeness and accuracy of data entry relies mainly on the enthusiasm of family practitioners. There are currently no agreed reference standards for reporting data quality in primary care and this limits measurement of data quality in electronic patient records". Weiskopf and Weng (17) noted that "data completeness of electronic 
health records is a real issue and there is no plausible solution available for looking at unstructured data". In their research they highlighted the fact that there is a need to introduce a method that assesses the unstructured clinical data for data completeness. In their article they also cited the research work carried out by Hogan and Wagner (18) which highlighted the fact that "data completeness ranged between $1.1 \%$ and $100 \%$, depending on the clinical concepts being studied". Furthermore, Majeed et al. (15) further state that, "development of methods and incentives for significantly improving the coding of clinical data and data quality in electronic primary care records remains a priority for health care information technology programs". Based on this statement the necessity of implementing an algorithm to assess data quality is extremely important. Here there is a belief that the solution to this problem does not lie in researching the methods and practices used in clinics (19).

Here it is important to mention that Bayesian networks have been used extensively over the years for the purpose of implementing machine learning in Clinical Decision Support Systems (CDSS). CDSS is defined by Wyatt and Spiegelhalter (20) as "an active knowledge system which uses two or more items of patient data to generate casespecific advice”. Several researchers have successfully used machine learning to advise clinical practices thereby improving patient satisfaction. This is especially true given the fact that clinical practices have to deal with a myriad of complexities including different types of diseases and disorders, patient lifestyles, nutrition, level of patient care, and other necessary factors that are of value when it comes to decision making. Nee and Hein (21) describe the use of Bayesian networks on cardiac tele-rehabilitation for patients with myocardial infarction. Here Bayesian networks have been used effectively to reduce the possibility of false alarms. A rule-based approach is used to for patient stabilization and alert generation. This confirms the idea that the use of Bayesian networks improves the efficiency of clinical decision support. Sesen et al. (22) illustrated the use of Bayesian networks in providing personalized treatment for lung cancer patients to improve the selection of the right treatment plan. The purpose of this analysis was to maximize the chances of patient survival. This research exemplifies the use of Bayesian networks in machine learning for the purpose of implementing expert systems to improve healthcare delivery. Yet et al. (23) describe the use of causal Bayesian networks in clinical decision making. Their research clearly indicates that the causal relationship between clinical intervention and observed results must be recorded to populate the required database. This information could be further used to identify the causal relationships that can be used to develop the Bayesian network. The network thus developed can be modified if required when there is a considerable change in the observed relationship. While these networks have been used in clinical interventions there is a critical necessity to use them in electronic health record systems to facilitate the necessary decision support on diagnosis.

Markov decision process is another heuristic approach used in healthcare decision support. Hauskrecht and Fraser (24) describe the use of a Markov decision process to plan the treatment of patients suffering from ischemic heart disease. In this research they use the Markov modeling approach to map diagnosis and treatment to outcome. Here the researchers attempt to address the uncertainty associated with diagnosis and treatment. The authors identify the fact that decision trees that are rigid may not be able to address the complex and dynamic nature of issues related to patients. Instead, they recommend the use of dynamic decision trees that allow flexibility to deal with the inherent complexity of the problem domain. Markov decision processes have also been used in deciding the right time for intervention by Magni et al. (25). This is especially true when medical interventions are carried out during life threatening situations. At times it could be necessary for physicians to optimize the intervention time using Markov decision processes with the intention of maximizing the benefit gained from intervention. One form of intervention to be considered would be drug administration where dynamic decision models could be of much use when compared to static decision models as described in (25). Collier et al. (26) have described the use of tags to represent Hidden Markov Models.

Models using ontology development is critical for implementing decision support while maintaining interoperability (27). Kataria and Juric (28) have illustrated the use of translating databases into ontologies and extracting necessary information from these ontologies for clinical decision support. It is to be noted that ontologies allow data to be self-descriptive in nature. Additionally, since these ontologies represent information in the form of hierarchy they are best suited for representing complex systems and situations. This is especially true if we believe Simon's statement (29) that "complexity takes the form of hierarchy". An important aspect of semantic web technologies is the representation of information to 
emphasize the required meaning between its individual components in a machine-actionable format. These meaningful representations could be characterized at multiple levels of computation. For example, representing information using RDF is the simplest with the highest level of computational guarantee. However, a higher computationally complex model such as forms of Web Ontology Language (OWL) may be best used for more complex relations at the cost of reduction in computational guarantee (28). Ontologies are many times associated with parsing Application Programming Interface (API) such as Clinical Text Analysis and Knowledge Extraction System (cTAKES) (30). cTAKES is an important API associated with the Unstructured Information Management Architecture (UIMA) Framework. The development of cTAKES started in 2006 under the leadership of computer scientists working with the Mayo Clinic.

Some of the well-known knowledge repositories include: (I) UMLS (31); (II) Bio2RDF; and (III) Systematized Nomenclature of Medicine-Clinical Terms (SNOMEDCT) (20). UMLS was developed by U.S. National Library of Medicine and is a tool that brings together biomedical and health related vocabularies in the form of a semantic network. This network could be used to develop decision support systems. UMLS is equipped with lexical tools used for annotating information which also helps in information extraction. Information is grouped together to form concepts. Bio2RDF (32) is a network of linked data pertaining to life sciences. This data is available to researchers in the form of RDF triples. These RDF triples could be parsed to perform reasoning and inferences. SNOMED-CT $(20,33)$ is a collection of medical terms used for representing them in a machine-actable format. SNOMED-CT encompasses synonyms, findings, and other necessary elements of clinical terms in a hierarchical format.

Based on the discussion provided the following conclusion on existing knowledge gaps could be derived in the following situations: (I) while there is abundance of general standards for representing electronic health records and interoperability there is a need for integrating heuristic models into the existing standards to advance the science of decision support for healthcare; and (II) there is a need to introduce data completeness algorithms for patient data using advances in decision science since this has not been done in the past. Any attempt to bridge these knowledge gaps will lead to a new area of science that deals with heuristics of decision making.

\section{A key observation in electronic health records affecting mHealth}

Our key contribution in this area lies in our solution to assess and analyze data incompleteness of electronic health records. The author first received the NSF award (CNS1735841) in seed funding from Fall 2018 UCF I-Corps Site program titled, "I-Corps: Data Completeness and Data Inconsistency in healthcare Data" and then received from award number 1928279 from the National I-Corps Teams program titled "Analyzing Data Completeness and Inconsistency to Reduce Misdiagnosis and Mitigate Re-imbursement Errors". In addition to this, the patent (US 10,790,049 B2) for measuring data completeness of electronic health records was issued by the United States patent office. This ground breaking research is not only applicable to regular monolithic systems that manage electronic health records but also to telehealth since many times patient data may be collected over the phone using an app. In this invention data completeness is defined using the Record Strength Score (RSS) score by first developing a concept map of an ideal complete electronic health record and then measuring the missing elements. This algorithm explained by Nasir et al. $(34,35)$ happens to be generic in nature and will lead to more sophisticated algorithms depending on the scenario, data lifecycle, data transmission process, or specialization under consideration.

It is to be noted that, outpatient clinics in rural areas lack clients, exacerbating the need for cost effective automated solutions to patient data problems. Based on the interviews conducted, some believe that data incompleteness can be resolved by full implementation of interoperability standards. However, most of the interviews showed that implementing interoperability between different electronic health record systems alone cannot solve this problem. Adibuzzaman et al. (36) points to this problem and addresses the need of having a continuously learning healthcare system. This further ascertains the need for implementing machine learning algorithms within the electronic health record systems. Further, while blockchain technologies can be of much help with regards to enhancing security of healthcare data it cannot be a solution for mistakes that can occur due to pitfalls in processes and tasks implemented within the healthcare data cycle.

The interviews conducted during the National I-Corps provided an opportunity for the author and his team members to approach many potential partners. Based on these interviews the potential partners who can benefit 


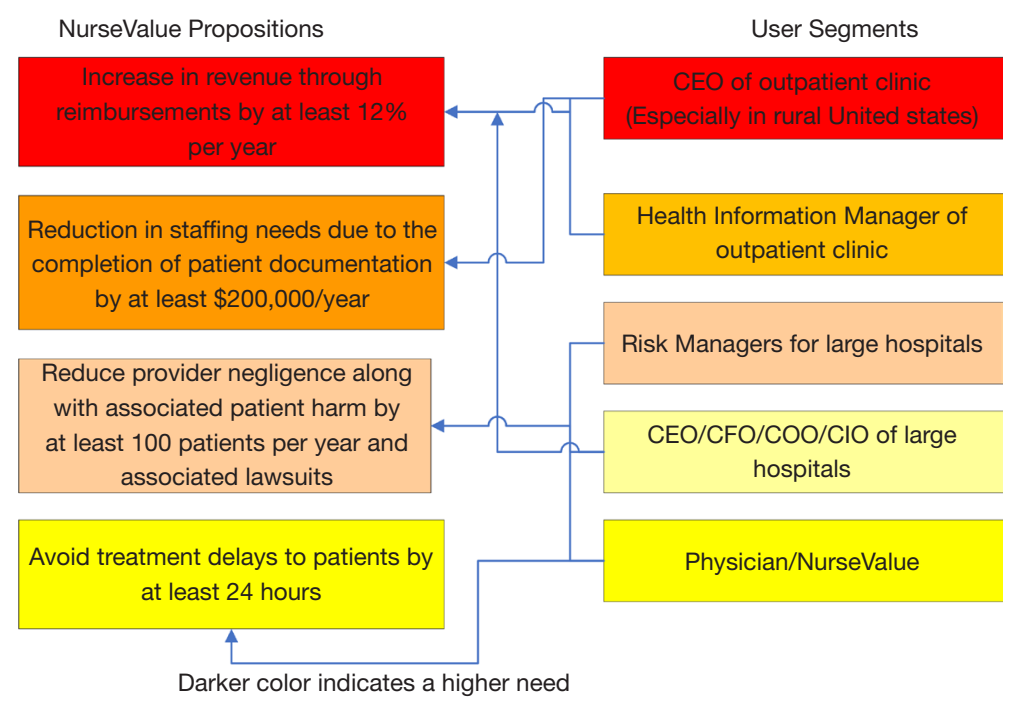

Figure 1 Value propositions and user segments for a data incompleteness solution. CEO, Chief Executive Officer; CFO, Chief Financial Officer; COO, Chief Operating Officer; CIO, Chief Information Officer.

and positively impact the society solving the problem of data incompleteness in electronic health records are: (I) vendors of the electronic health record systems; (II) owners of outpatient clinics in rural areas; (III) organizations that provide data solutions; and (IV) health care process improvement organizations as indicated in Figure 1. This is especially true for vendors who have not yet captured a large section of the market and would like to expand their outreach into the market by having a cutting-edge solution that can be useful in providing better services to healthcare providers. As discussed before outpatient clinics in rural areas (37) have much to gain from this invention. The invention can be used as an add-on to the existing electronic health record solutions. This can be achieved by using the algorithm in a cloud-based application service with a monthly or annual subscription fee. Additionally, organizations that provide data solutions to the healthcare providers (38) other than the electronic health record vendors can be a potential partner and will enable the invention to be used as an application service with large healthcare providers. Last but not the least, organizations that work with large healthcare providers in improving their processes termed as "Healthcare Process Improvement Organizations" can benefit by using this invention in identifying pitfalls in the lifecycle of patient data (39-42).

The algorithm used here takes into account three important variables: (I) the existence or non-existence of an attribute value in an electronic health record; (II) the importance weight for each of these attributes; and (III) the sum of these weights. It is common knowledge that the electronic health records are stored in the form of relational databases. These databases contain rows and columns identified as tuples and attributes by computer scientists. The algorithm analyzes the presence or absence of important attributes in tuples. A particular attribute may be important due to two reasons: (I) diagnostic reasoning; and (II) reimbursement for services provided by the provider. The value proposition illustrated in Figure 1 focuses more on the latter. This is mainly because it has been noticed that healthcare providers have been going out of business due to dearth of timely reimbursements.

\section{Conclusion and future work}

Overall, the article discussed the some of the key complexities and an important problem associated with electronic health records (43). The discussion presented in the article proceeded with the critical contribution made by the author and his colleagues made towards the specific problem of data incompleteness applied to electronic health records (44) that applies to the delivery of mHealth. Some of the key contributions provided by the article are as follows: (I) a discussion on the complexities of technologies associated with electronic health records adding to the body of knowledge in this area; (II) identification of data incompleteness as a critical problem that needs further 
investigation.

It is perhaps worthwhile exploring the use of right type of technologies that will mitigate the problem of data incompleteness; although, many of the factors that lead to data incompleteness are human factors that may not be influenced by a particular technology. Here the author believes that the research conducted in this area of science is still very embryonic in nature and much more needs to be achieved. Additionally, the discussion presented in this article will motivate researchers and developers of technology (45) to seriously consider working on some of the critical problem areas (46) that need to be targeted mentioned in this article.

\section{Acknowledgments}

The author would like to thank the Guest Editors Dr. Donna Slovensky and Dr. Donna Malvey for providing the opportunity to contribute to the special series.

Funding: The research work was funded by NSF Award \#1928279; I-Corps: Data Completeness and Inconsistency Analysis Platform.

\section{Footnote}

Provenance and Peer Review: This article was commissioned by the Guest Editors (Donna J. Slovensky and Donna M. Malvey) for the series "mHealth: Innovations on the Periphery" published in mHealth. The article has undergone external peer review.

Conflicts of Interest: The author has completed the ICMJE uniform disclosure form (available at https://mhealth. amegroups.com/article/view/10.21037/mhealth-21-39/ coif). The series "mHealth: Innovations on the Periphery" was commissioned by the editorial office without any funding or sponsorship. The author has no other conflicts of interest to declare.

Ethical Statement: The author is accountable for all aspects of the work in ensuring that questions related to the accuracy or integrity of any part of the work are appropriately investigated and resolved.

Open Access Statement: This is an Open Access article distributed in accordance with the Creative Commons Attribution-NonCommercial-NoDerivs 4.0 International
License (CC BY-NC-ND 4.0), which permits the noncommercial replication and distribution of the article with the strict proviso that no changes or edits are made and the original work is properly cited (including links to both the formal publication through the relevant DOI and the license). See: https://creativecommons.org/licenses/by-nc-nd/4.0/.

\section{References}

1. Gurupur VP, Tanik MM. A system for building clinical research applications using semantic web-based approach. J Med Syst 2012;36:53-9.

2. Jain GP, Gurupur V, Faulkenberry ED. Artificial Intelligence Based Student Learning Evaluation Tool. Proceedings of the IEEE Educon 2013;751-6.

3. Jain GP, Gurupur V, Schroeder JL, et al. Artificial Intelligence-Based Student Learning Evaluation: A Concept Map-Based Approach for Analyzing a Student's Understanding of a Topic. IEEE Transactions on Learning Technologies 2014;7:267-79.

4. Lucas PJ, van der Gaag LC, Abu-Hanna A. Bayesian networks in biomedicine and health-care. Artif Intell Med 2004;30:201-14.

5. Dong M, He D. Hidden Semi-Markov Model-based Methodology for Multi-sensor Equipment Health Diagnosis and Prognosis. European Journal of Operational Research 2007;178:858-78.

6. Groner R, Groner M, Bishchof WF. Editors. Methods of Heuristics. Taylor and Francis Group, New York; 2014.

7. Sarker RA, Abbass HA, Newton C. Heuristic \& Optimization for Knowledge Discovery. Idea Group Publishing, London; 2002.

8. Kahneman D, Slovic P, Tversky A. Editors. Judgement under uncertainty: Heuristics and biases. Cambridge University Press, Cambridge, UK; 2001:2-47.

9. Michalski RS, Carbonell JG, Mitchell TM. Machine Learning: An Artificial Intelligence Approach, SpringerVerlag, Berlin; 1984:11.

10. Marewski JN, Gigerenzer G. Heuristic decision making in medicine. Dialogues Clin Neurosci 2012;14:77-89.

11. Charles C, Gafni A, Whelan T. Shared decision-making in the medical encounter: what does it mean? (or it takes at least two to tango). Soc Sci Med 1997;44:681-92.

12. Gurupur V, Sakoglu U, Jain GP, et al. Semantic requirements sharing approach to develop software systems using concept maps and information entropy: A Personal Health Information System example. Adv Eng Softw 2014;70:25-35. 
13. Orgun MA, Meyer T. Introduction to the special issue on advances in ontologies. Expert Systems 2008;25:175-8.

14. Hunink MGM, Weinstein MC, Wittenberg E, et al. Decision Making in Health and Medicine: Integrating Evidence and Values, 2nd Edition, Cambridge University Press, Cambridge, United Kingdom; 2014.

15. Majeed A, Car J, Sheikh A. Accuracy and completeness of electronic patient records in primary care. Fam Pract 2008;25:213-4.

16. Nasir A, Gurupur V, Liu X, et al. Managing Healthcare Patient Data Using the Data Completeness Analysis Package (DCAP). Proceedings of SDPS 2015 Conference; 2015:108-11.

17. Weiskopf NG, Weng C. Methods and dimensions of electronic health record data quality assessment: enabling reuse for clinical research. J Am Med Inform Assoc 2013;20:144-51.

18. Hogan WR, Wagner MM. Accuracy of data in computer-based patient records. J Am Med Inform Assoc 1997;4:342-55.

19. Rittenhouse DR, Shortell SM. The patient-centered medical home: will it stand the test of health reform? JAMA 2009;301:2038-40.

20. Wyatt J, Spiegelhalter D. Evaluating Medical Expert Systems: What To Test, And How? Knowledge Based Systems in Medicine: Methods, Applications and Evaluation; 1991. doi: 10.1007/978-3-662-08131-0_22.

21. Nee O, Hein A. Clinical Decision Support with Guidelines and Bayesian Networks. Advances in Decision Support Systems, INTECH, Croatia; 2010:342.

22. Sesen MB, Nicholson AE, Banares-Alcantara R, et al. Bayesian networks for clinical decision support in lung cancer care. PLoS One 2013;8:e82349.

23. Yet B, Perkins Z, Marsh W, et al. Towards a method of building causal Bayesian networks for prognostic decision support. Probabilistic Problem Solving in Biomedicine 2011;107:107-48.

24. Hauskrecht M, Fraser H. Planning treatment of ischemic heart disease with partially observable Markov decision processes. Artif Intell Med 2000;18:221-44.

25. Magni P, Quaglini S, Marchetti M, et al. Deciding when to intervene: a Markov decision process approach. Int J Med Inform 2000;60:237-53.

26. Collier N, Nobata C, Tsujii J. Extracting the names of genes and gene products with a hidden Markov model. Proceedings of the 18th conference on Computational linguistics 2000:201-7.

27. Chen D, Doumeingts G. European initiatives to develop interoperability of enterprise applications-basic concepts, framework and roadmap, Annual reviews in Control 2003;27:153-62.

28. Kataria P, Juric R. Sharing e-health information through ontological layering, Proceedings of the 43rd Hawaii International Conference on Systems Sciences; 2010. doi:10.1109/HICSS.2010.338.

29. Simon HA. The architecture of complexity. Proceedings of the American Philosophical Society 1962;106:467-82.

30. Savova GK, Masanz JJ, Ogren PV, et al. Mayo clinical Text Analysis and Knowledge Extraction System (cTAKES): architecture, component evaluation and applications. J Am Med Inform Assoc 2010;17:507-13.

31. Bodenreider O. The Unified Medical Language System (UMLS): integrating biomedical terminology. Nucleic Acids Res 2004;32:D267-70.

32. Belleau F, Nolin MA, Tourigny N, et al. Bio2RDF: towards a mashup to build bioinformatics knowledge systems. J Biomed Inform 2008;41:706-16.

33. Stearns MQ, Price C, Spackman KA, et al. SNOMED clinical terms: overview of the development process and project status. Proc AMIA Symp 2001;662-6.

34. Nasir A, Gurupur V, Liu X. A New Paradigm to Analyze Data Completeness of Patient Data. Appl Clin Inform 2016;7:745-64.

35. Nasir A, Liu X, Gurupur V, et al. Disparities in patient record completeness with respect to the health care utilization project. Health Informatics J 2019;25:401-16.

36. Adibuzzaman M, DeLaurentis P, Hill J, et al. Big data in healthcare - the promises, challenges and opportunities from a research perspective: A case study with a model database. AMIA Annu Symp Proc 2018;2017:384-92.

37. Hart LG, Salsberg E, Phillips DM, et al. Rural health care providers in the United States. J Rural Health 2002;18 Suppl:211-32.

38. Wang Y, Kung L, Byrd TA. Big data analytics: Understanding its capabilities and potential benefits for healthcare organizations. Technol Forecast Soc Change 2018;126:3-13.

39. Thor J, Lundberg J, Ask J, et al. Application of statistical process control in healthcare improvement: systematic review. Qual Saf Health Care 2007;16:387-99.

40. Kaplan HC, Provost LP, Froehle CM, et al. The Model for Understanding Success in Quality (MUSIQ): building a theory of context in healthcare quality improvement. BMJ Qual Saf 2012;21:13-20.

41. Hatler CW, Mast D, Corderella J, et al. Using evidence and process improvement strategies to enhance healthcare 
outcomes for the critically ill: a pilot project. Am J Crit Care 2006;15:549-55.

42. Kulkarni SA, Pannu JS, Koval AV, et al. A Brief Analysis of Key Machine Learning Methods for Predicting Medicare Payments Related to Physical Therapy Practices in the United States. Information 2021;12:57-75.

43. Gurupur V, Shelleh M. Machine Learning Analysis for Data Incompleteness (MADI): Analyzing the Data Completeness of Patient Records Using a Random Variable Approach to Predict the Incompleteness of Electronic Health Records. IEEE Access 2021;9:95994-6001.

doi: $10.21037 /$ mhealth-21-39

Cite this article as: Gurupur VP. Key observations in terms of management of electronic health records from a mHealth perspective. mHealth 2022;8:18.
44. Gurupur V. A Review on Advances in Design and Development of Complex Adaptive Systems for Healthcare Using Concept Maps. Health Technol 2021;5:8.

45. Clark S, Elswick S, Gabriel M, et al. Transitions of Care: A Patient-Centered Perspective of Health Information Systems that Support Post-Acute Care. Journal of Integrated Design and Process Science 2016;20:95-110.

46. Gurupur V, Kulkarni SA, Liu X, et al. Analysing the Power of Deep Learning Techniques Over the Traditional Methods Using Medicare Utilization and Provider Data. Journal of Experimental \& Theoretical Artificial Intelligence 2018;31:99-115. 